\title{
The MAL protein is crucial for proper membrane condensation at the ciliary base, which is required for primary cilium elongation
}

\author{
Elena Reales ${ }^{1, *}$, Miguel Bernabé-Rubio ${ }^{1, *}$, Javier Casares-Arias ${ }^{1}$, Carles Rentero², Jaime Fernández-Barrera ${ }^{1}$, \\ Laura Rangel ${ }^{1}$, Isabel Correas ${ }^{1}$, Carlos Enrich ${ }^{2}$, Germán Andrés ${ }^{3}$ and Miguel A. Alonso ${ }^{1, \ddagger}$
}

\begin{abstract}
The base of the primary cilium contains a zone of condensed membranes whose importance is not known. Here, we have studied the involvement of MAL, a tetraspanning protein that exclusively partitions into condensed membrane fractions, in the condensation of membranes at the ciliary base and investigated the importance of these membranes in primary cilium formation. We show that MAL accumulates at the ciliary base of epithelial MDCK cells. Knockdown of MAL expression resulted in a drastic reduction in the condensation of membranes at the ciliary base, the percentage of ciliated cells and the length of the cilia, but did not affect the docking of the centrosome to the plasma membrane or produce missorting of proteins to the pericentriolar zone or to the membrane of the remaining cilia. Rab8 (for which there are two isoforms, Rab8A and Rab8b), IFT88 and IFT20, which are important components of the machinery of ciliary growth, were recruited normally to the ciliary base of MAL-knockdown cells but were unable to elongate the primary cilium correctly. MAL, therefore, is crucial for the proper condensation of membranes at the ciliary base, which is required for efficient primary cilium extension.
\end{abstract}

KEY WORDS: Primary ciliogenesis, Centrosome, Condensed membranes, MAL

\section{INTRODUCTION}

The primary cilium is a single appendage that projects from the cell surface in most vertebrate cells. It is made up of a ciliary membrane that surrounds a microtubule-based structure, termed the axoneme, which is nucleated from the older of the two centrioles in the centrosome. Primary cilia sense a diverse range of environmental signals in the extracellular milieu and relay these to the cell body through surface receptors specifically localized on the ciliary membrane (Gerdes et al., 2009; Goetz and Anderson, 2010; Ishikawa and Marshall, 2011). Defects in primary cilium functioning are associated with a growing list of human developmental and degenerative disorders, collectively referred to as ciliopathies, that simultaneously affect many organs in the body (Hildebrandt et al., 2011; Novarino et al., 2011).

\footnotetext{
${ }^{1}$ Department of Cell Biology and Immunology, Centro de Biología Molecular Severo Ochoa, Consejo Superior de Investigaciones Científicas and Universidad Autónoma de Madrid, Cantoblanco, Madrid 28049, Spain. ${ }^{2}$ Departament de Biologia Cel·lular, Immunologia i Neurociències, Institut d'Investigacions Biomèdiques August Pi i Sunyer (IDIBAPS), Facultat de Medicina, Universitat de Barcelona, Barcelona 08036, Spain. ${ }^{3}$ Electron Microscopy Unit, Centro de Biología Molecular Severo Ochoa, Consejo Superior de Investigaciones Científicas and Universidad Autónoma de Madrid, Cantoblanco, Madrid 28049, Spain.

*These authors contributed equally to this work

${ }^{\ddagger}$ Author for correspondence (maalonso@cbm.csic.es)
}

Received 27 October 2014; Accepted 30 April 2015
By assessing the degree of lipid condensation, it has been found that the base of cilia is more condensed than the surrounding apical membrane of epithelial Madin-Darby canine kidney (MDCK) cells (Vieira et al., 2006). Possible functions postulated for the condensed membranes at the ciliary base include that it acts to facilitate the docking of the basal body to the plasma membrane, that it modulates the elongation of the ciliary membrane, that it has a structural role or that it functions as a barrier for controlling the composition of the ciliary membrane (Reiter and Mostov, 2006; Vieira et al., 2006). Previous studies have revealed that knockdown(KD) of phosphatidylinositol-4-phosphate adaptor protein-2 (FAPP2, also known as PLEKHA8) alters the condensation of the apical membrane and reduces primary cilium formation suggesting that there is a link between apical membrane condensation and primary ciliogenesis (Vieira et al., 2006). However, the importance and role of the condensed membranes specifically found at the ciliary base in the process of primary cilium assembly are still unclear.

MAL, a 17-kDa tetraspanning membrane protein, partitions into detergent-insoluble membrane fractions (Cheong et al., 1999; Puertollano et al., 1999), which are enriched in condensed membranes also known as membrane rafts (Gaus et al., 2003; Lingwood and Simons, 2010). MAL was initially characterized as a component of the specialized machinery that regulates the rate of transport of proteins to the apical surface of MDCK cells (Cheong et al., 1999; Puertollano et al., 1999). MAL levels are important for primary ciliogenesis in MDCK cells (Takiar et al., 2012; Torkko et al., 2008), but the role of MAL in this process has remained unexplored. In this work, we show that MAL, which localizes at the base of the primary cilium, is important for membrane condensation at the ciliary base. Using MAL KD cells, we characterized the stage at which the ciliogenesis process is impacted by reduced condensation of membranes at the ciliary base. We found that the centrosome docked efficiently to the plasma membrane and that the ciliary base still controlled the access of membrane proteins and recruited machinery for primary cilium growth but, without proper membrane condensation, the ciliary base was unable to elongate the cilium efficiently. MAL and periciliary condensed membranes, therefore, appear to be required for efficient functioning of the machinery of primary cilium growth.

\section{RESULTS}

\section{MAL expression is induced with cell confluence}

Renal epithelial MDCK cells assemble a primary cilium when they reach confluence and become quiescent. To establish whether MAL expression is regulated by cell confluence, MDCK cells were grown for different times and the levels of MAL were analyzed by immunoblotting. The formation of primary cilia and the expression of the adhesion protein E-cadherin, which is known to be upregulated with increased cell-cell contacts, were examined in 
parallel. MAL levels increased with cell confluence similar to E-cadherin levels and concomitantly with the number of ciliated cells, whereas the expression of caveolin-1, which was used as the control for the experiment, remained unaltered (Fig. 1A,B). The increase of MAL levels upon cell confluence correlated with the upregulation of MAL mRNA, as determined by quantitative realtime PCR analysis (qRT-PCR) (supplementary material Fig. S1A). In conclusion, MAL expression accompanies cell polarization, including primary cilium formation.

\section{MAL is crucial for efficient primary cilia formation}

MDCK cells segregate apical and basolateral domains and assemble a primary cilium at the center of the apical surface. To investigate the requirement for MAL in primary ciliogenesis we compared primary cilium formation in MDCK cells transfected with a control small interfering RNA (siRNA) (siC) or with siRNAs (sil MAL and si2 MAL) targeting the sequences surrounding the AUG translation initiation site or the $3^{\prime}$ untranslated region, respectively, of canine MAL mRNA. The extent of endogenous MAL KD was $90-95 \%$ in the case of si1 MAL and $70 \%$ in the case of si2 MAL, as assessed by immunoblotting (Fig. 2A,B, supplementary material Fig. S1B). MAL KD produced an important decrease in the number of cilia and in the length of the remaining cilia in these cells (Fig. 2C-E, supplementary material Fig. S1C). Given that sil MAL was more effective than si2 MAL, we used sil MAL in all subsequent experiments. We used MDCK cells that express the intact coding sequence of human MAL mRNA (denoted MDCK-MAL cells) as an additional control. This sequence exhibited one mismatch with sil MAL, and therefore the expression of human MAL in these cells was predicted to be less sensitive to sil MAL than is endogenous dog MAL, as indeed proved to be the case (Fig. 2A,B). Cilia number and ciliary length were not affected in MDCK-MAL cells in which the endogenous protein was knocked down with sil MAL (Fig. 2C-E). In summary, MAL, whose expression is induced by the time primary ciliogenesis starts, is essential for normal primary ciliogenesis.

The docking of the centrosome to the center of the apical membrane, as visualized with GFP-centrin (Fig. 3A) and the presence of the centriole subdistal and distal appendage protein marker Odf2 at the centrosome were not perturbed in MAL KD cells (Fig. 3B). The distribution of basolateral ( $\beta$-catenin) and tight junction (ZO-1, also known as TJP1) markers were also unaffected (Fig. $3 \mathrm{C}$ ), as was the periciliary ring delimiting the ciliary base visualized by galectin-3 staining (Fig. 3D). Consistent with our confocal microscopic studies, electron microscopic analysis showed that MAL KD cells exhibited stunted cilia compared with control cells (Fig. 4A, supplementary material Fig. S2). In addition, the older centriole, which is referred to as the basal body when it is docked to the plasma membrane to form a cilium, was correctly apposed to the plasma membrane and its transition fibers were apparently normal in MAL KD cells (Fig. 4, supplementary material Fig. S2). No apparent accumulation of vesicles was detected in the vicinity of the basal body in MAL KD cells (Fig. 4A, supplementary material Fig. S2). The analysis of sequential longitudinal sections confirmed that cilia in MAL KD cells were shorter than in control cells (supplementary material Fig. S2). Examination of sequential cross-sections below the transition fibers showed that the basal body had the typical triplet microtubule structure in MAL KD cells (Fig. 4B). The analysis of sequential cross-sections above the basal body revealed that the appearance of microtubule singlets occurred close to the basal body in the case of MAL KD cells (Fig. 4B). In summary, Figs 3, 4 and supplementary material Fig. S2 indicate that the effect of MAL silencing in primary ciliogenesis is not a consequence of defects in centrosome structure, basal body docking, periciliary ring assembly, fusion of transport vesicles or cell polarization.

\section{MAL is important for membrane condensation at the ciliary base}

We next analyzed the distribution of MAL relative to the primary cilium. As the current antibodies available to canine MAL are not of use in immunofluorescence studies, we used our anti-human MAL mAb 6D9 and the MDCK-MAL cell clone previously used in the rescue-of-function experiments (Fig. 2C-E). Consistent with previous observations (Martin-Belmonte et al., 1998), the 6D9 mAb recognized exogenous human MAL, but not endogenous canine protein (Fig. 5A). As revealed by double labeling with antibodies to MAL and $\alpha$-tubulin, exogenous MAL was found to concentrate predominantly at the base of the cilium relative to the surrounding apical membrane in moderately confluent cell cultures (Fig. 5A, middle panel; Fig. 5B) and also at the primary cilium at high cell confluency (Fig. 5A, bottom panel; Fig. 5C). The distribution of MAL indicates that it could play a role at the ciliary base during primary ciliogenesis and at the primary cilium.

The packing density of biological membranes can be directly measured using the Laurdan fluorescent membrane dye (Gaus et al., 2006). The Laurdan dye penetrates the cell membrane and aligns parallel to the phospholipids (Bagatolli et al., 2003), undergoing a shift in its peak emission wavelength from $500 \mathrm{~nm}$ in fluid membranes to $440 \mathrm{~nm}$ in ordered membranes. A normalized ratio of the two emission regions, given by the general polarization
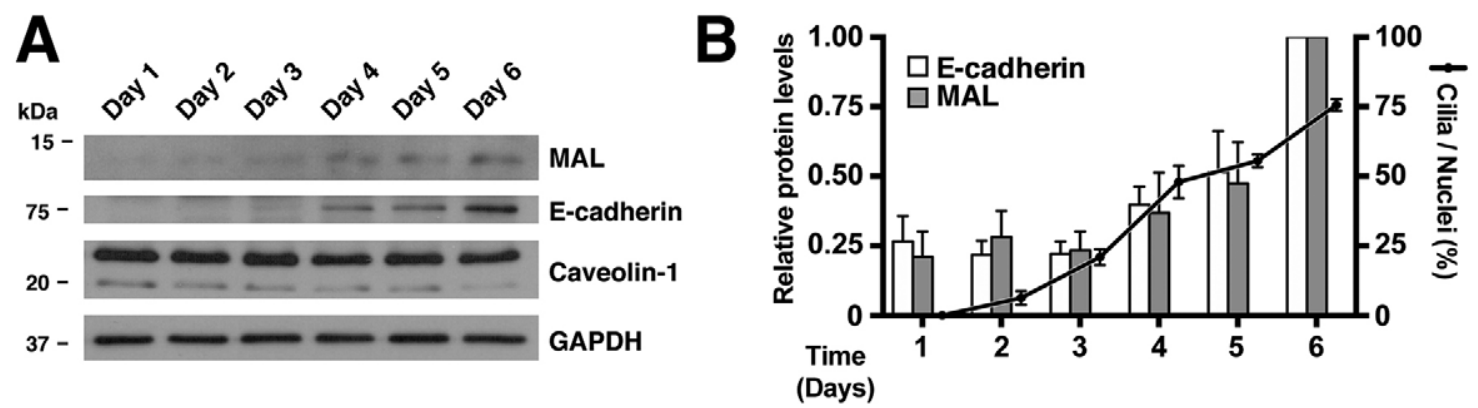

Fig. 1. MAL expression is induced with cell confluence and regulates primary ciliogenesis. (A,B) Extracts of MDCK cells grown for different times were immunoblotted for the indicated proteins. The levels of MAL and E-cadherin were quantified and normalized to those of glyceraldehyde 3-phosphate dehydrogenase (GAPDH), which was used as loading control (left $y$-axis). The number of cilia was determined in parallel experiments (right $y$-axis). The histogram illustrates the levels of MAL and the number of cilia relative to the values on day $6(B)$. Data are the mean \pm s.e.m. from at least three independent experiments. 
A

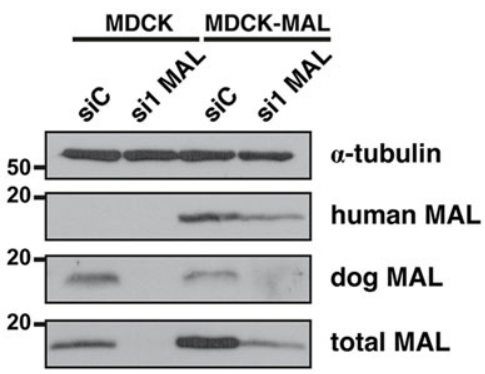

C

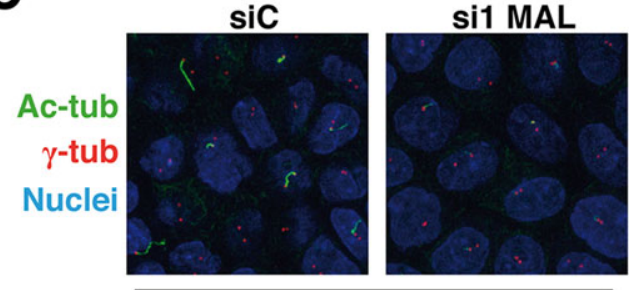

MDCK

D

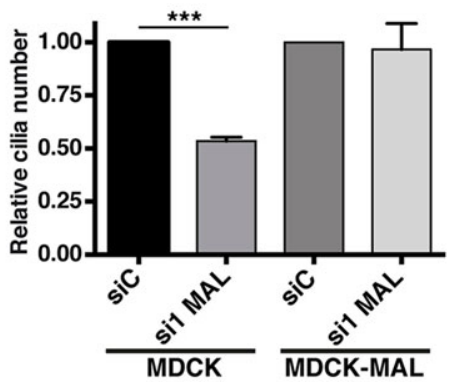

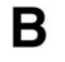

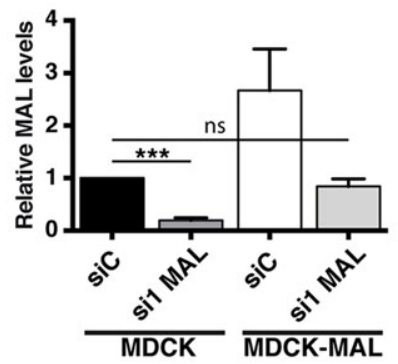

E

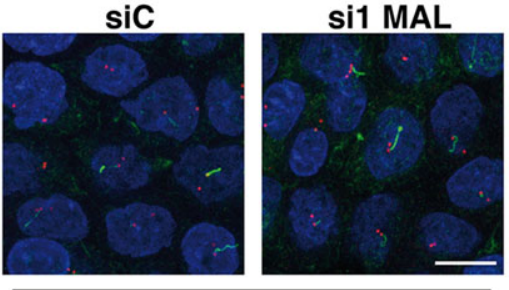

MDCK-MAL

Fig. 2. MAL regulates primary ciliogenesis. (A-E) Control or MDCK cells stably expressing human MAL (MDCK-MAL) were transfected with the indicated siRNA. Cell extracts were immunoblotted for $\alpha$-tubulin or for human (mAb 6D9), dog (mAb 2E5) or total (goat polyclonal) MAL, as indicated (A) The intensity of the MAL bands obtained with the antibody to total MAL was quantified. The values obtained for MAL were corrected using those of $\alpha$-tubulin and represented relative to those in contro cells (B). Cells grown in Transwell inserts for $72 \mathrm{~h}$ were stained for acetylated-tubulin (Ac-tub), $\gamma$ tubulin and nuclei $(C)$. The number of primary cilia was determined and expressed relative to that in control cells transfected with $\mathrm{siC}$ (D). The length of the cilia was measured and the values were grouped in the indicated size ranges. The results represent the percentage of cilia in each range relative to the total number of cilia present in each condition $(E)$. Scale bar: $10 \mu \mathrm{m}$. Data in B, D and $E$ are the mean \pm s.e.m. from at least three independent experiments. ${ }^{*} P<0.05$; ${ }^{* \star *} P<0.001$; ns, not significant.

index provides a relative measure of membrane order. This experimental approach was previously used to demonstrate the existence of condensed membranes at the ciliary base of MDCK cells (Vieira et al., 2006). To investigate whether MAL regulates membrane condensation at this important zone, we stained the cells with Laurdan and measured membrane order at the ciliary

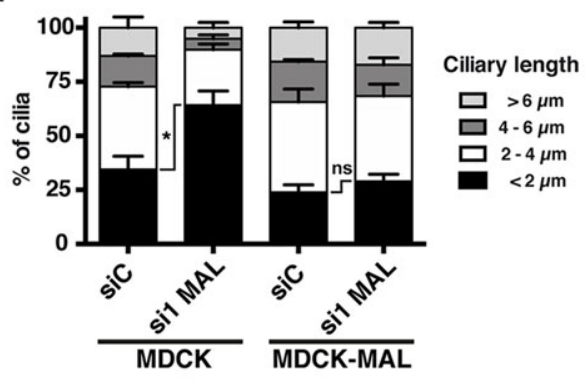

A

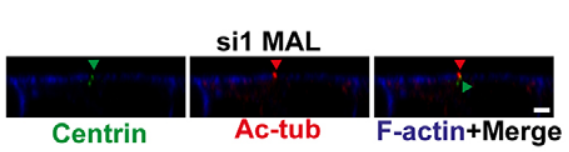

B

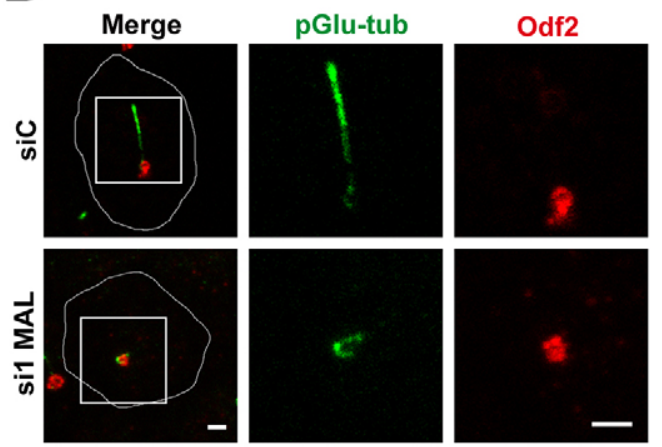

C
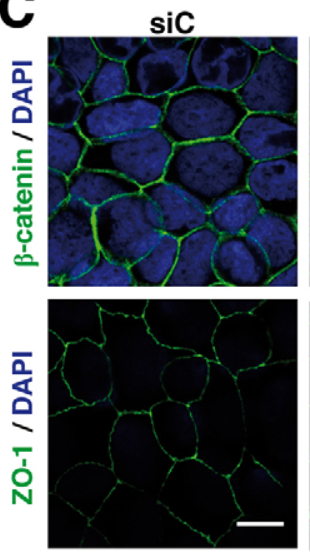

D

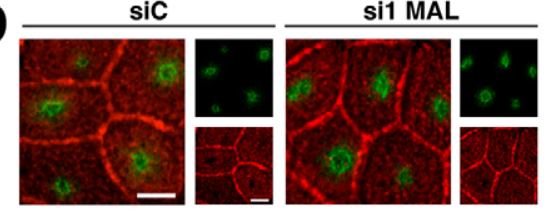

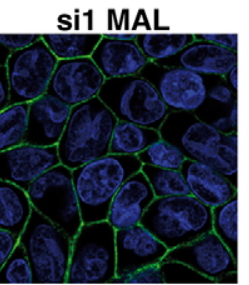
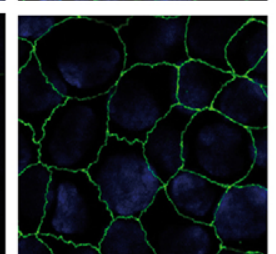

si1 MAL

$\frac{4}{6=}$

base, which was visualized by galectin-3 labeling. Membrane condensation at the ciliary base was dramatically diminished in MAL KD cells, whereas it was not significantly affected in the rest of the apical membrane (Fig. 5D,E). This result indicates that MAL expression is crucial for membrane condensation at the ciliary base.

Galectin-3/F-actin

Fig. 3. Effect of MAL knockdown on the distribution of a range of protein markers. (A) MAL KD cells stably expressing GFPcentrin (green arrowhead) grown on Transwell inserts were stained for acetylated tubulin (Ac-tub, red arrowhead) and F-actin. The $x z$ projection shows that the centrosome was correctly positioned at the center of the apical membrane. Scale bar: $2 \mu \mathrm{m}$. (B) Cells were stained for the subdistal and distal appendage marker Odf2 (red) and polyglutamylated tubulin (pGlu-tub, green), which stains the axoneme. The white line indicates the cell contour. The merged image is shown on the left. The right panels show the separate stainings. Scale bars: $3 \mu \mathrm{m}$.

(C) Cells were grown in Transwell inserts for $72 \mathrm{~h}$. Cells were labeled with DAPI to visualize the nuclei and stained for $\beta$-catenin or ZO-1, as indicated. Scale bar: $10 \mu \mathrm{m}$.

(D) Cells were stained for galectin- 3 and F-actin. The merged image is shown on the left. The right panels show the separate stainings. Scale bar: $3 \mu \mathrm{m}$. 
A
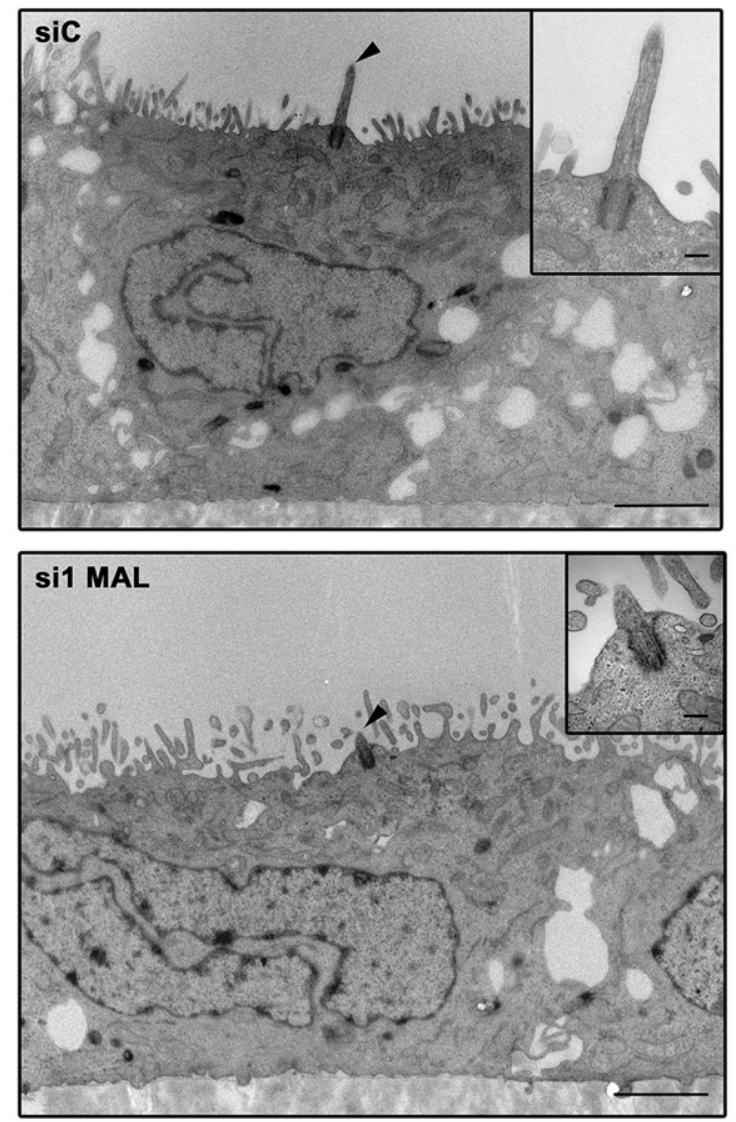

B
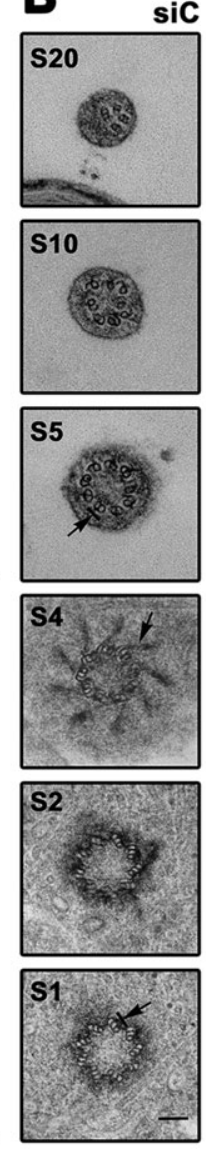

sic

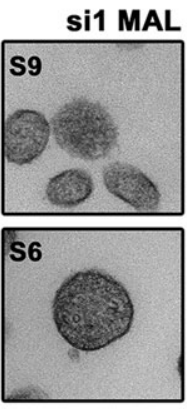

s5
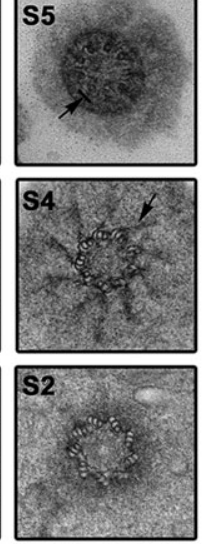

Fig. 4. Ultrastructural analysis of the primary cilium in control and MAL-knockdown cells. MDCK cells grown on Transwell filters for 5 days were fixed, embedded in resin and sectioned orthogonally to or in parallel with the supporting substrate.

(A) Representative image of a primary cilium in control and MAL KD cells. Note that the primary cilium (arrowhead), which appears longitudinally sectioned, is shorter in MAL-KD cells than in control cells. The insets show the primary cilium at higher magnification.

(B) Representative images of a cross-sectioned primary cilium of control and MAL KD cells. The sections are numbered from the basal body (S1 to S4) to the tip of the cilium (S5 onwards). Note that although the cilium ends prematurely the overall ultrastructure of the basal body and the beginning of the cilium in MAL KD cells are similar to those in control cells. Arrows in $\mathrm{S} 1$ point to the microtubule triplets of the basal body, in S4 to the transition filaments (S4), and in S5 to the axoneme doublets. Scale bars: $1 \mu \mathrm{m}(\mathrm{A}) ; 50 \mathrm{~nm}(\mathrm{~B}$, insets in $\mathrm{A})$.

\section{Machinery of primary cilium growth is efficiently recruited to the ciliary base in MAL knocked-down cells}

One of the functions postulated for the condensed membranes of the ciliary base is that it serves as a fence to separate the ciliary membrane from the surrounding apical membrane proteins (Reiter and Mostov, 2006; Vieira et al., 2006). If this were the case, disruption of the condensed membranes at the ciliary base would allow the entry to the pericentriolar zone and the ciliary membrane of proteins that are normally excluded. We compared the distribution of several apical membrane proteins normally excluded from the ciliary base and ciliary membrane in control and MAL KD cells (Fig. 6). We did not detect in MAL KD cells a significant increase in the access to the pericentriolar zone of the $\mathrm{p} 75$ neurotrophin receptor (also known as NGFR) fused to GFP (p75-GFP) (Kreitzer et al., 2003), Smoothened (Smo), a seven-pass transmembrane protein that functions in the Hedgehog signaling pathway (Chen et al., 2002), endogenous podocalyxin (Meder et al., 2005) or exogenous CD59, chosen as a representative of glycophosphatidylinositol (GPI)-anchored proteins (Lisanti et al., 1990; Lisanti and Rodriguez-Boulan, 1990). In summary, Fig. 6 shows that the condensed membranes of the ciliary base do not appear to function as a general barrier to the entry of membrane proteins into the primary cilium.

The small GTPases Rab8 (for which there are two isoforms, Rab8a and Rab8b) and Rab10 (Knodler et al., 2010) and the machinery for intraflagellar transport (IFT) are necessary for primary cilium growth (Rosenbaum and Witman, 2002). Stunted or absent cilia, as seen in MAL KD cells, have been described in cells with deficient expression of Rab8 (Westlake et al., 2011), which regulates exocytosis of ciliary components to the ciliary base, or IFT machinery, which transports proteins along the ciliary membrane (Rosenbaum and Witman, 2002). It is of note that Rab8 and two components of the IFT machinery, IFT88 and IFT20 (Follit et al., 2006; Pazour et al., 2000), distributed along the primary cilium and the pericentriolar region in control cells, whereas the three proteins localized to the pericentriolar region of MAL KD cells lacking a primary cilium (Fig. 7A-C). Smo resides outside the primary cilium under basal conditions and translocates to the primary cilium in response to Hedgehog or the Smo agonist (Milenkovic et al., 2009). Whereas Smo-GFP distributed along the ciliary membrane in control cells after agonist stimulation, we found that it accumulated at the pericentriolar region in MAL KD cells (Fig. 7D). The quantitative analysis indicated a marked increase in the presence of all the markers analyzed at the pericentriolar area in MAL $\mathrm{KD}$ cells (Fig. 7E). The remaining cilia of MAL KD cells were as positive as those of control cells for the four ciliary markers (Fig. 7F). The results illustrated in Fig. 7 rule out the possibility that the impairment of ciliary growth observed in MAL KD cells is due to a defect in the recruitment of Rab8 or IFT machinery to the ciliary base, or to a general defect in protein targeting to the periciliary compartment, and point to a failure in primary cilia elongation caused by the reduced condensation of membranes at the ciliary base.

\section{DISCUSSION}

A zone of highly condensed membranes of unknown importance exists at the ciliary base of epithelial MDCK cells (Vieira et al., 2006). We observed that depletion of MAL, a protein that selectively partitions into condensed membrane fractions, reduced membrane condensation at the ciliary base and inhibited primary ciliogenesis. We used MAL KD cells to test several hypothesized 
A
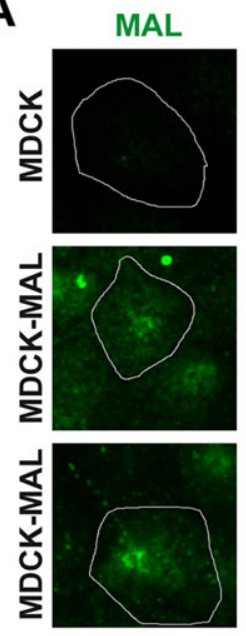

D
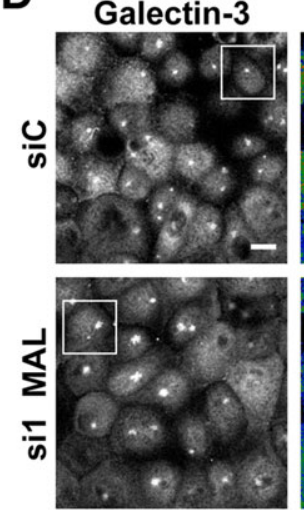

a-tub
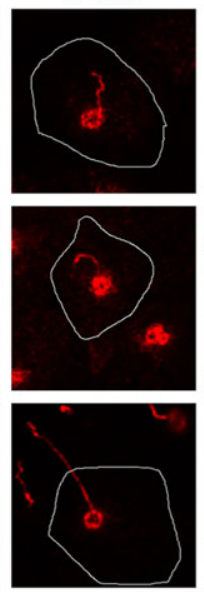

GP
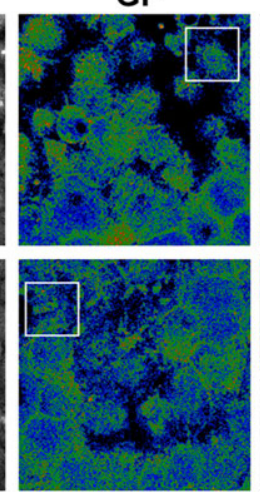

Merge

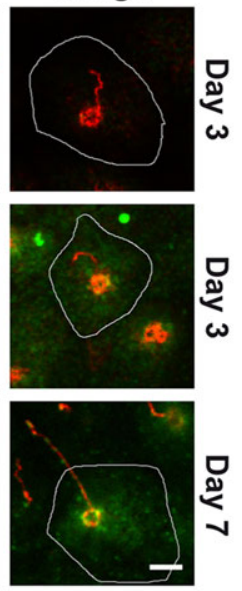

,
B

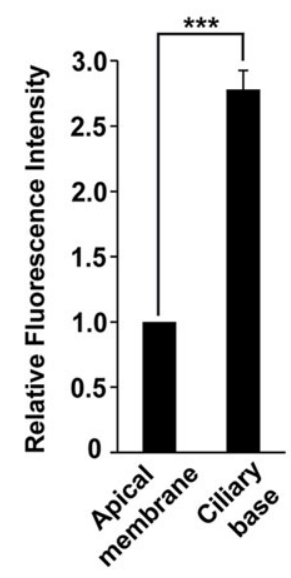

C

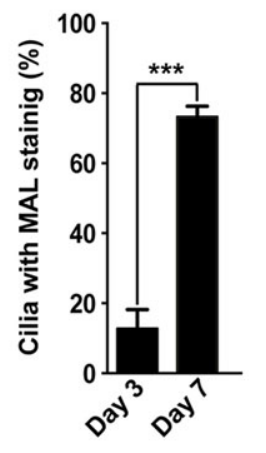

E

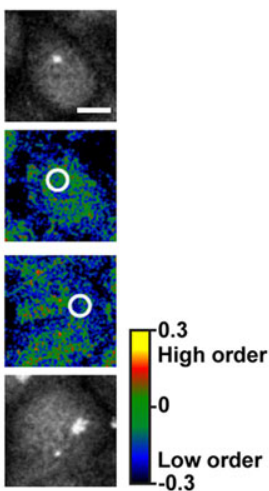

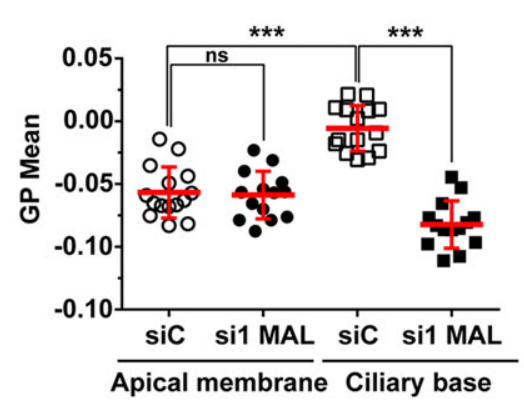

Fig. 5. MAL concentrates at the base of the primary cilium and is important for membrane condensation at the ciliary base. (A-C) Control (top panels) or MDCK-MAL cells (middle and bottom panels) grown on Transwell inserts for 3 or 7 days were immunolabeled for exogenous MAL and $\alpha$-tubulin. The white line indicates the cell periphery. Scale bar: $3 \mu \mathrm{m}(\mathrm{A})$. The intensity of MAL staining at day 3 was quantified in rings enclosing the ciliary base. The values are expressed relative to the mean of three identical rings chosen at random from the surrounding apical surface (B). The percentages of cilia immunolabeled for MAL at day 3 and day 7 were determined $(C)$. (D,E) Control or MAL KD cells were stained with Laurdan and immunolabeled for galectin-3. Laurdan intensity images were converted into general polarization (GP) images and pseudocolored using the scale on the right to represent low-to-high general polarization values. Scale bar: $10 \mu \mathrm{m}$. An enlargement of the boxed region is shown on the right. Scale bar: $3 \mu \mathrm{m}$. The general polarization value corresponding to rings enclosing the membrane area delimited by galectin-3 (ciliary base) or to random zones of the same size in the apical membranes (apical) was calculated and represented (E). 15 images were used and 10-20 ciliary bases were evaluated. Data in B, C and E are the mean \pm s.e.m. from three independent experiments (individual data points are also shown in $\mathrm{E}) .{ }^{* * *} P<0.001$; ns, not significant.

roles for condensed membranes at the ciliary base (Reiter and Mostov, 2006; Vieira et al., 2006). We concluded that MAL and these specialized membranes are not essential for docking the basal body to the plasma membrane, periciliary ring assembly or fusion of transport vesicles to the base zone. Nor do condensed membranes have general functions in excluding non-ciliary proteins from the ciliary base. Instead, interaction with the cytoskeleton (Francis et al., 2011), or physical barriers based on nucleoporins (Kee et al., 2012), septins (Hu et al., 2010) or on a complex that includes proteins disrupted in ciliopathies (Chih et al., 2011) are involved in regulating the protein composition of the primary cilium. The observation that, despite the presence of protein trafficking machinery at the ciliary base, MAL KD cells were unable to form primary cilia efficiently, and that even those that were formed were shorter than those of control cells, suggest that MAL and condensed membranes are necessary for primary cilium elongation.

Protein trafficking machinery is required for primary cilium assembly and maintenance. On the one hand, the IFT machinery aided by microtubule motors is responsible for protein transport along the cilium. On the other hand, Rab GTPases and the exocyst complex (Heider and Munson, 2012) orchestrate the assembly of the primary cilium through a network of interactions that results in a supply of membranes and ciliary growth (Das and Guo, 2011; Lim et al., 2011; Yoshimura et al., 2007; Zuo et al., 2009). Centrosome distal appendage proteins link the mother centriole with Rab GTPases and the exocyst through the direct interaction of Odf2 with Rab8 (Yoshimura et al., 2007), and the association of Odf2 with Sec15 (also known as EXOC6 in mammals), a subunit of the exocyst, mediated by centriolin (Hehnly et al., 2012). IFT20, which is also present in the Golgi, helps transport vesicular material to the ciliary base (Follit et al., 2006). We examined the presence of Odf2 at the centrosome and the distribution of IFT20, IFT88 and Rab8 and found that all of them were present at the centrosome zone regardless of MAL expression. Therefore, the ciliogenesis defect observed in MAL KD cells was not due to defective recruitment of these components. MAL specifically associates with condensed membrane or raft-enriched fractions and mediates the apical transport of the influenza virus hemagglutinin (Cheong et al., 
A
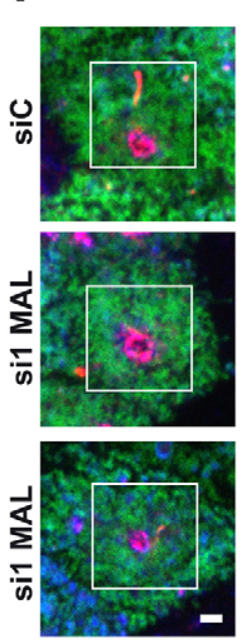

B
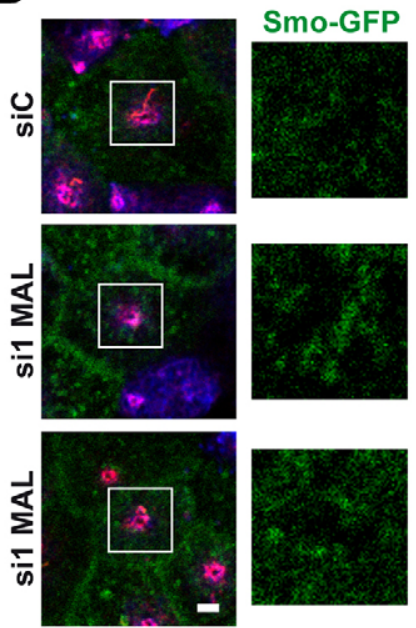

E

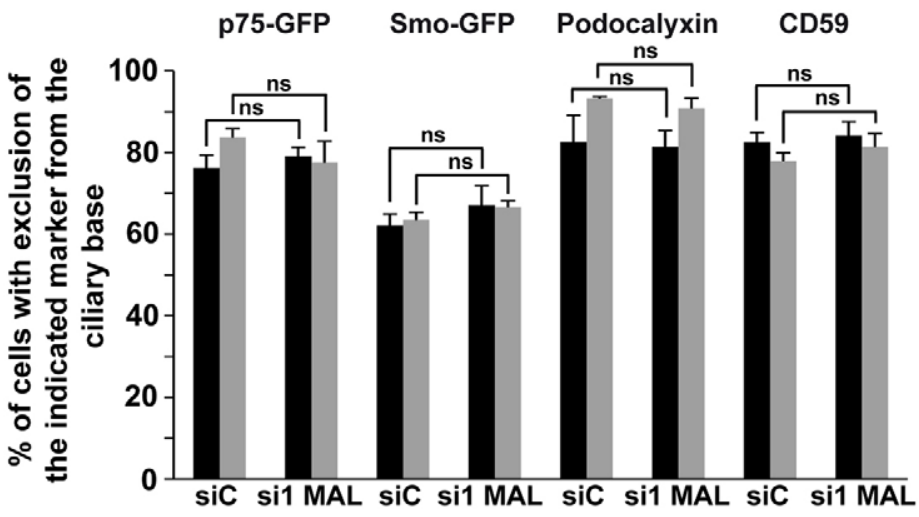

C

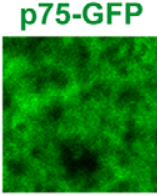

$\alpha-$ tub

Galec-3
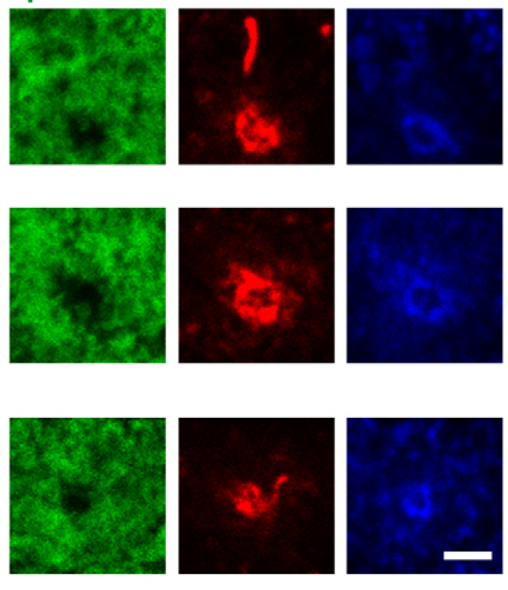

D
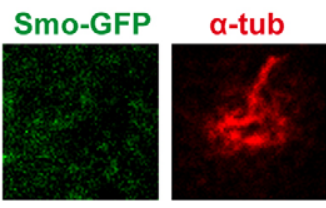

Galec-3
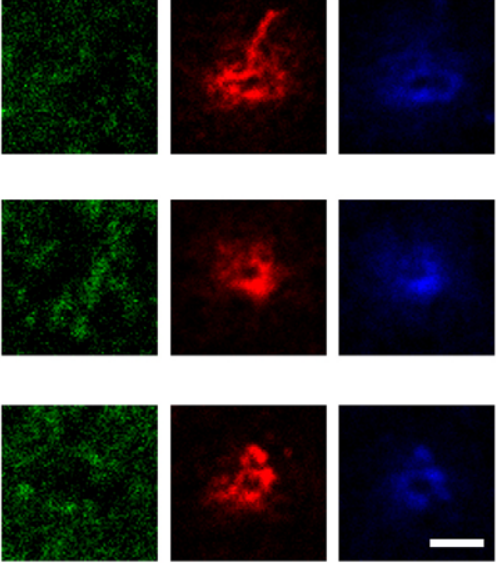

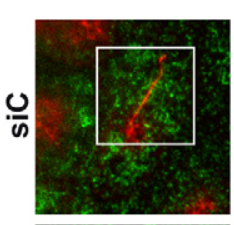

Podocalyxin $\alpha$-tub
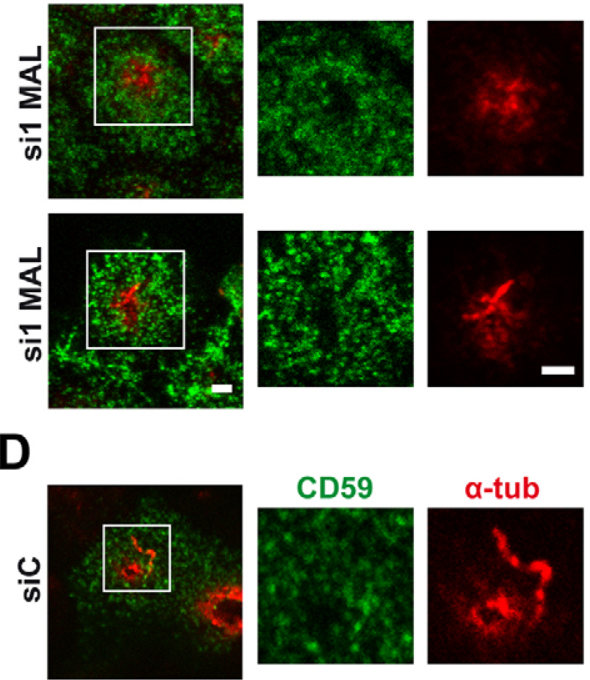

a-tub
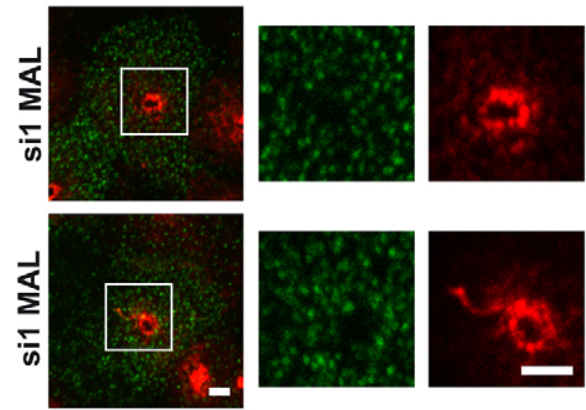

non-ciliated cells

ciliated cells

Fig. 6. Exclusion of different apical membrane proteins from the ciliary base. (A-E) Control (top panels) or MAL KD cells (middle and bottom panels) grown on Transwell inserts were labeled for galectin-3 and/or tyrosinated $\alpha$-tubulin and p75-GFP (A), Smo-GFP in cells without Smo agonist treatment (B), endogenous podocalyxin (C), or exogenous CD59 (D). MAL KD cells without (middle panels) and with (bottom panels) a primary cilium are shown. Note that in A,B both galectin-3 and tyrosinated $\alpha$-tubulin delimit the periciliary ring. Scale bars: $2 \mu \mathrm{m}$. The histogram shows the percentage of cells showing exclusion of the indicated markers from the ciliary base in cells without and with a primary cilium (E). Data are the mean $\pm s . e . m$. from three independent experiments. ns, not significant.

1999; Puertollano et al., 1999), a protein cargo associated with raftenriched membrane fractions. We examined the extent of condensation of the membranes at the ciliary base and found striking differences between control and MAL KD cells. Given that the knockdown of endogenous MAL was quite effective it would be expected that it affects the entire cell population to a greater or lesser extent and, therefore, that the loss of membrane condensation would impair primary cilium formation in the majority of the MAL KD cells. In the case of MAL KD cell population with a primary cilium, the reduction of membrane condensation was probably lower 
A
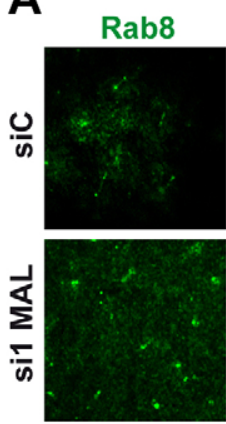

C IFT20
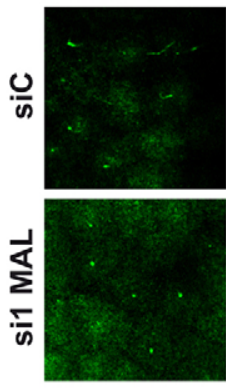

$\mathbf{E}$
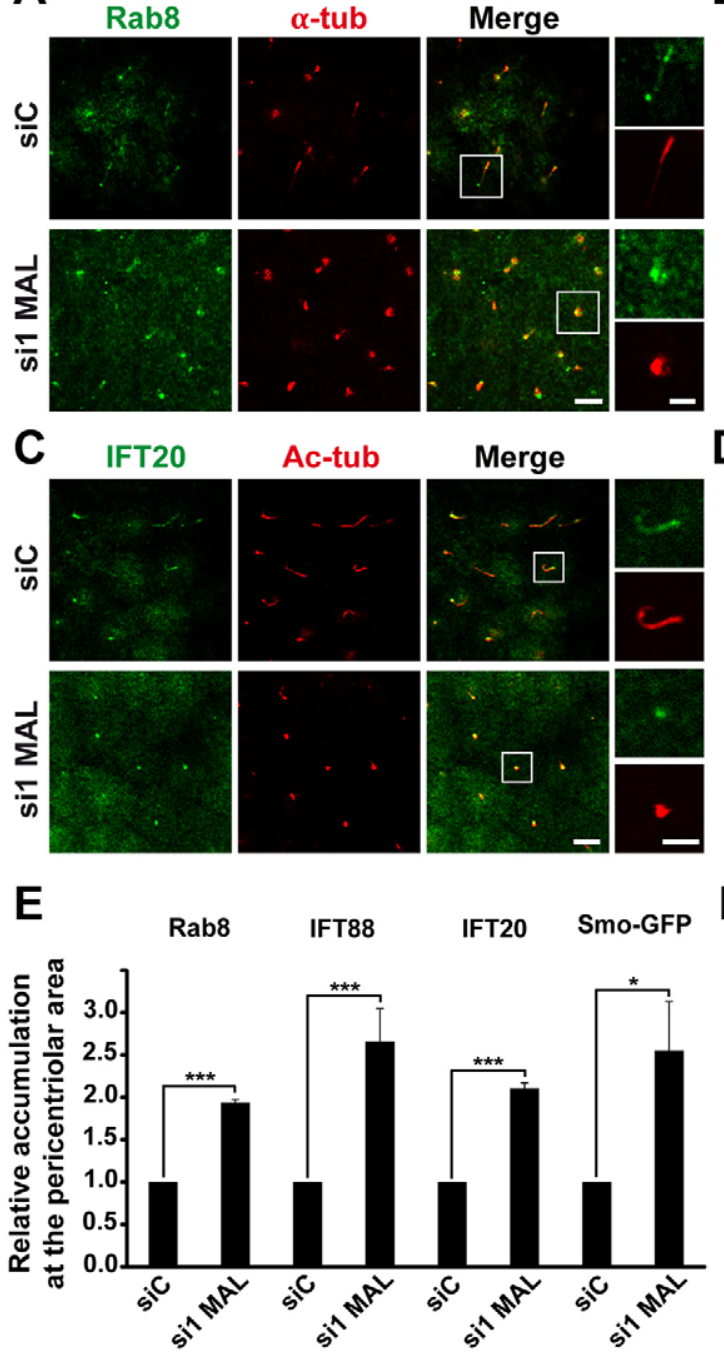

Ac-tub

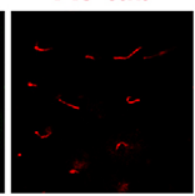

Merge
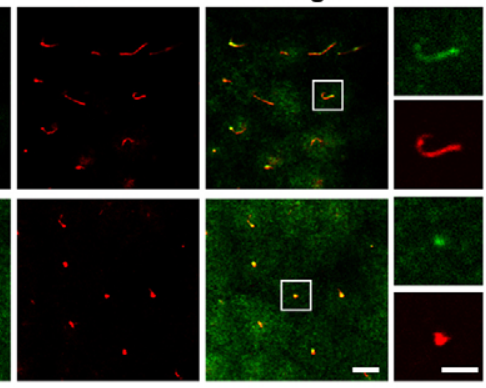

B
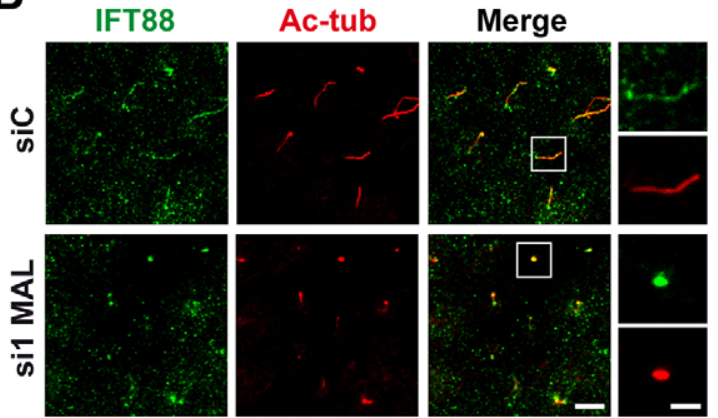

D Smo-GFP

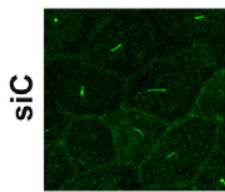

Ac-tub

Merge
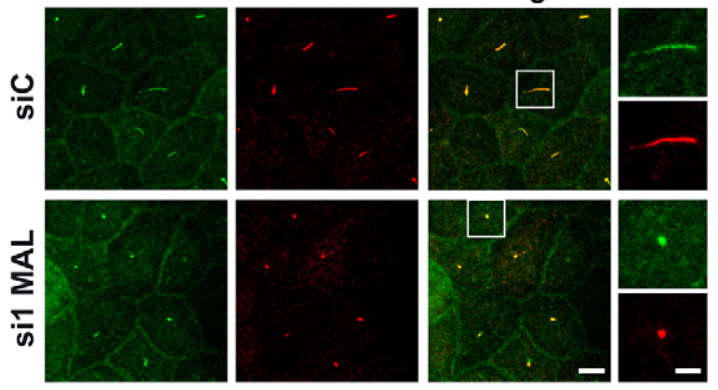

$\mathbf{F}$

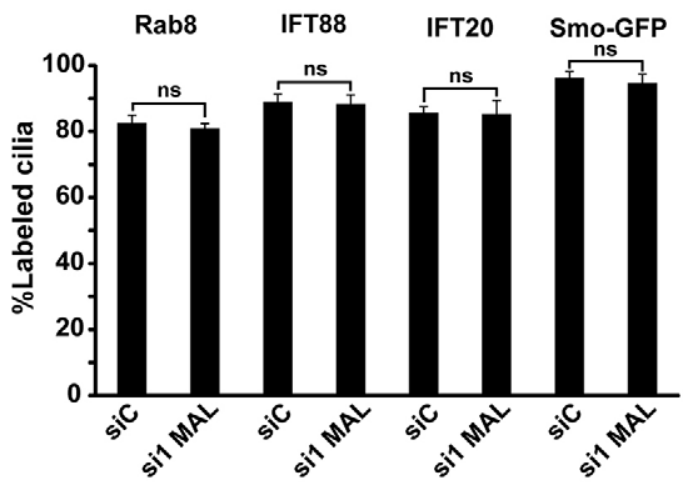

Fig. 7. The impairment of ciliary growth in MAL KD cells is not due to general defects in the recruitment of ciliary extension protein machinery.

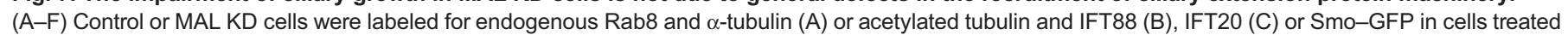
with $100 \mathrm{nM}$ Smo agonist for $24 \mathrm{~h}$ (D). Scale bars: $5 \mu \mathrm{m}$ (main panels), $2 \mu \mathrm{m}$ (enlargements). The percentage of MAL KD cells where the indicated markers

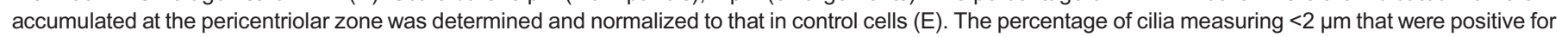
the indicated markers was measured in control cells and in the remaining cilia of MAL KD cells. Data in E,F are the mean \pm s.e.m. from three independent experiments $\left({ }^{*} P<0.05 ;{ }^{* * *} P<0.001\right.$; ns, not significant).

allowing the formation of primary cilia with varying sizes. Therefore, we find it is plausible that there is a threshold of membrane condensation at the ciliary base below which the process of ciliogenesis is totally blocked, whereas above which it takes place to a varying extent, depending on the degree of condensation. The treatment of MDCK cells with fumonisin B1, an inhibitor of ceramide biosynthesis, impairs both the percentage of ciliated cells and the length of the remaining cilia (Wang et al., 2009). Given that ceramide is a lipid component of condensed membranes, the effect of fumonisin B1 might be due to a reduction in membrane condensation at the ciliary base. Previous studies have revealed that silencing of FAPP2, another protein involved in apical transport (Vieira et al., 2005), decreases the percentage of ciliated cells in MDCK cells (Vieira et al., 2006). FAPP2 KD cells showed an increase of apical membrane condensation and a $12 \%$ reduction in the content of condensed domains of the apical surface (Vieira et al., 2006). Given that the possible effect of FAPP2 KD on the condensation of membranes at the base of the primary cilium was not addressed, we do not know whether FAPP2 is also important for proper membrane condensation at the ciliary base. Given the role of MAL in the transport of raft-associated molecules (Anton et al., 2011; Cheong et al., 1999; Puertollano et al., 1999), it is plausible that MAL affects membrane condensation at the ciliary base through the supply of raft membranes, although a direct role of MAL in the organization of raft lipids is also feasible (Magal et al., 2009). One interesting possibility is that the condensed membranes at the periciliary region are required for efficient assembly of machinery for primary cilium growth. The role of MAL would be reminiscent to that in $\mathrm{T}$ cells, in which MAL transports vesicles containing raft-associated Lck and regulates membrane condensation at the immunological synapse (Anton et al., 2011). This similarity offers one additional example of the proposed parallels between the primary cilium and the immunological synapse (Angus and Griffiths, 2013; Finetti and Baldari, 2013). 
Among the many abnormalities associated with ciliopathies, renal cystic diseases are the most common (Hildebrandt and Otto, 2005; Watnick and Germino, 2003), making research on primary cilium formation in renal epithelial cells, such as MDCK cells, particularly important, especially given that the mechanism of the ciliogenesis process is cell-type- and tissue-dependent (Sorokin, 1962). Our results indicate that MAL, which is expressed in MDCK cells by the time that primary ciliogenesis commences, concentrates at the ciliary base, and is crucial for the proper condensation of membranes at the ciliary base, which is required for efficient primary cilia elongation.

\section{MATERIALS AND METHODS \\ Reagents}

The mouse monoclonal antibody (mAb) 6D9 specific to human MAL and the rat mAb 2E5 specific to canine MAL have been previously described (Millán and Alonso, 1998; Puertollano et al., 1999). The source of commercial antibodies to the indicated proteins used was as follows: $\gamma$-tubulin (rabbit polyclonal, T3559; mouse mAb IgG1, clone GTU-88, T6557), total $\alpha$-tubulin (mouse mAb IgG1, clone DM1A, T9026), acetylated tubulin (mouse mAb IgG2b, clone 6-11B-1, T7451) and IFT20 (rabbit polyclonal, HPA021376) were from Sigma; galectin3 (rat mAb IgG2a, clone M3/38, 125401) was from Biolegend; polyglutamylation modification (mouse $\mathrm{mAb}$ IgG1, clone GT335, AG-20B-0020) was from Adipogen; tyrosinated $\alpha$-tubulin (rat $\mathrm{mAb}$ IgG2a, clone YL1/2, MA1-80017) was from Thermo Scientific; caveolin-1 (rabbit polyclonal, 610059) and Rab8 (mouse mAb IgG2b, 610844) were from BD Transduction Laboratories; ZO-1 (rat mAb IgG1, R26.4C) was from the Developmental Studies Hybridoma Bank; CD59 (mouse mAb IgG2b, ab9183) was from Abcam; podocalyxin (mouse $\mathrm{mAb}$ ) was a gift from George K. Ojakian (Downstate Medical Center, New York, NY); IFT88 (rabbit polyclonal, 13967-AP) was from Proteintech; $\beta$-catenin (rabbit polyclonal, sc-7199), Odf2 (goat polyclonal, sc-23134) and MAL (goat polyclonal antibody, sc-46171) were from Santa Cruz Biotechnology; glyceraldehyde 3-phosphate dehydrogenase (mouse mAb IgG1, clone 6C5, AM4300) was from Life Technologies. Fluorescent phalloidin and secondary antibodies conjugated to Alexa-Fluor-488, -594 or -647 were from Life Technologies. Horseradish peroxidase (HRP)-conjugated secondary antibodies were from Jackson ImmunoResearch. Smo agonist [N-Methyl-N'-(3-pyridinylbenzyl)-N'-(3-chlorobenzo[b]thiophene-2carbonyl)-1,4-diaminocyclohexane] was from Merck Millipore.

\section{Cell culture, siRNA, DNA constructs and transfection conditions}

Epithelial canine MDCK II cells were grown in MEM supplemented with $5 \%$ fetal bovine serum (Sigma-Aldrich) at $37^{\circ} \mathrm{C}$ in a $5 \% \mathrm{CO}_{2}$ atmosphere. $10^{6}$ cells were electroporated with $20 \mathrm{nM}$ of siRNA-negative control Hi GC, si1 MAL (5'-GCUGGGUGAUGUUUGUGUCUGUGUU-3') or si2 MAL (5'-GGUGUUAUGUUUACUCUCCCAUAUA-3') stealth-RNAi ${ }^{\text {TM }}$ (Life Technologies) using Amaxa equipment run with the L-005 program. Cells were cultured on 35-mm-diameter dishes for $24 \mathrm{~h}$ and then grown on 12-mm polycarbonate membranes of $0.2 \mu \mathrm{m}$ pore size (Transwell, Costar, Inc., Cambridge, MA) for $72 \mathrm{~h}$. Knockdown was verified by immunoblotting $96 \mathrm{~h}$ after transfection. The DNA constructs expressing Smo-GFP (Addgene plasmid 25395) (Chen et al., 2002) and GFP-centrin (Addgene plasmid 29559) (Salisbury et al., 2002) were gifts from Philip Beachy (Stanford School of Medicine, Stanford, CA) and Jeffrey Salisbury (Mayo Clinic, Rochester, MN), respectively. The plasmids expressing the p75GFP and CD59, were generous gifts from Enrique Rodriguez-Boulan (Cornell University, New York, NY) and Václav Horejsi (Institute of Molecular Genetics, Prague, Czech Republic), respectively. The DNA construct expressing intact human MAL was made in the pCDNA3.1 Zeo vector (Life Technologies). MDCK cell clones stably expressing Smo-GFP, GFP-centrin or human MAL were generated by transfection of the appropriate plasmid and selection with $1 \mathrm{mg} / \mathrm{ml} \mathrm{G}-418$ (Smo-GFP and GFP-centrin) or $0.75 \mu \mathrm{g} / \mathrm{ml}$ zeocin (MAL) (Life Technologies), respectively. The resulting clones were screened by immunofluorescence and immunoblotting.

\section{Immunofluorescence microscopic analysis}

Cells were fixed in formalin for $15 \mathrm{~min}$, rinsed, and treated with $10 \mathrm{mM}$ glycine in PBS for $5 \mathrm{~min}$ to quench the aldehyde groups. Cells were then washed, permeabilized or not with $0.1 \%$ Triton X-100 in PBS at room temperature for $5 \mathrm{~min}$, rinsed, incubated with $3 \%(\mathrm{w} / \mathrm{v}) \mathrm{BSA}$ for $15 \mathrm{~min}$, and incubated with the primary antibody. After $1 \mathrm{~h}$ at room temperature, cells were washed and incubated with the appropriate fluorescent secondary antibody. For double-labeling experiments, the same procedure was repeated for the second primary antibody. Cells were mounted in coverslips using ProLong Gold antifade reagent (Invitrogen). Images were obtained using a LSM 710 confocal microscope (Carl Zeiss) with a $63 \times$ objective. Brightness and contrast were optimized with Photoshop software (Adobe). Quantifications were performed using Image J software.

\section{Laurdan cell microscopy}

Confluent MDCK cells grown on glass coverslips were stained with $50 \mu \mathrm{M}$ Laurdan (6-dodecanoyl-2-dimethylaminonaphthalene; Life Technologies) for $30 \mathrm{~min}$ at $37^{\circ} \mathrm{C}$ (Gaus et al., 2003), fixed with formalin and immunostained for galectin-3. Laurdan imaging was performed with a TCS SP5 inverted confocal microscope (Leica) equipped with a near infrared laser (Mai Tai Broad Band 710-990 nm) and a HCX PL APO CS lambda blue $63 \times$ oil objective (1.4 NA). Confocal A555 signal (excitation $561 \mathrm{~nm}$; emission $570-620 \mathrm{~nm}$ ) was recorded followed by two-photon Laurdan images acquired at $800 \mathrm{~nm}$ excitation and simultaneously collected emission ranges at $400-460 \mathrm{~nm}$ and $470-530 \mathrm{~nm}$. Generalized polarization (GP) images were calculated pixel by pixel from the Laurdan intensity images according to the equation: $\mathrm{GP}=\left(I_{(400-460)}-I_{(470-530)}\right) /\left(I_{(400-460)}+I_{(470-530)}\right)$ (Owen et al., 2011). A total of 10-20 circular areas per condition, corresponding to the ciliary base, were selected on the basis of galectin-3 staining. Generalized polarization values were non-linearly fitted to a Gauss distribution using a custom-built macro in ImageJ (Rentero et al., 2008).

\section{Electron microscopy}

For transmission electron microscopy analysis, cells grown on Transwell filters were fixed with $4 \%$ paraformaldehyde and $2 \%$ glutaraldehyde for $90 \mathrm{~min}$ at room temperature. Cell samples were then processed for embedding in Epoxy, TAAB 812 Resin (TAAB Laboratories, Berkshire, UK) according to standard procedures. Orthogonal and parallel (from the bottom to the top of the cell) 80 -nm-thick ultrathin sections were stained with saturated uranyl acetate and lead citrate by standard procedures. Samples were examined at $80 \mathrm{kV}$ in a Jeol JEM-1010 (Tokyo, Japan) electron microscope. Pictures were taken with a TemCam-F416 $(4 \mathrm{~K} \times 4 \mathrm{~K})$ digital camera (TVIPS, Gauting, Germany).

\section{qRT-PCR}

Total RNA from MDCK cells was purified using RNeasy (Qiagen). MAL and E-cadherin mRNA levels were quantified by qRT-PCR procedures using the Super Script III First-Strand Synthesis SuperMix kit (Life Technologies, PN 11752250) and the qPCR FAST Sybr Green PCR Master Mix kit (Applied Biosystems, PN 4367659) in an ABI 7900HT equipment. The results were normalized with respect to the expression of $\beta$-actin, caveolin-1 and glyceraldehyde 3-phosphate dehydrogenase mRNA in the same samples. Data were analyzed with GenEX software.

\section{Immunoblotting}

Samples were subjected to SDS-PAGE and transferred onto Immobilon-P membranes (Millipore). After blocking with 5\% non-fat dried milk powder and $0.05 \%$ Tween- 20 in PBS, blots were incubated with the appropriate antibodies for $1 \mathrm{~h}$. After several washings, blots were incubated for $30 \mathrm{~min}$ with secondary antibodies coupled to horseradish peroxidase. The signal was visualized with ECL chemiluminescence detection reagent (Thermo Scientific). Band intensities were quantified using Image $\mathbf{J}$ software and results were expressed relative to the control condition. 


\section{Statistical analysis}

Data are expressed as the mean \pm s.e.m. of at least three independent experiments. Unless otherwise indicated, statistical significance was determined from a one-sample Student's $t$-test, calculated with GraphPad Prism software.

\section{Acknowledgements}

The expert technical advice of the personnel of the Optical and Confocal Microscopy, Electron Microscopy, and Genomics Units of the CBMSO is gratefully acknowledged. We thank Milagros Guerra for her invaluable assistance in the preparation of the EM sections.

\section{Competing interests}

The authors declare no competing or financial interests.

\section{Author contributions}

E.R and M.B.-R. performed most of the experiments; J.C.-A. analyzed MAL expression and contributed to some of the experiments; J-F.-B. and L.R. participated in some of the experiments; C.E and C.R. performed and analyzed the Laurdan microscopic analysis; I.C. designed and interpreted some of the experiments and participated in the writing of the manuscript; G.A. performed the EM analysis; M.A.A. designed and analyzed the experiments and wrote the manuscript.

\section{Funding}

This work was supported by the Ministerio de Economía y Competitividad, Spain [grant numbers BFU2012-32532 and CONSOLIDER COAT CSD2009-00016 to M. A.A.]. G.A. was supported by the Amarouto Program for senior researchers from the Comunidad Autónoma de Madrid.

Supplementary material

Supplementary material available online at

http://jcs.biologists.org/lookup/suppl/doi:10.1242/jcs.164970/-/DC1

\section{References}

Angus, K. L. and Griffiths, G. M. (2013). Cell polarisation and the immunological synapse. Curr. Opin. Cell Biol. 25, 85-91.

Anton, O. M., Andrés-Delgado, L., Reglero-Real, N., Batista, A. and Alonso, M. A. (2011). MAL protein controls protein sorting at the supramolecular activation cluster of human T lymphocytes. J. Immunol. 186, 6345-6356.

Bagatolli, L. A., Sanchez, S. A., Hazlett, T. and Gratton, E. (2003). Giant vesicles, Laurdan, and two-photon fluorescence microscopy: evidence of lipid lateral separation in bilayers. Methods Enzymol. 360, 481-500.

Chen, J. K., Taipale, J., Cooper, M. K. and Beachy, P. A. (2002). Inhibition of Hedgehog signaling by direct binding of cyclopamine to Smoothened. Genes Dev. 16, 2743-2748.

Cheong, K. H., Zacchetti, D., Schneeberger, E. E. and Simons, K. (1999). VIP17/ MAL, a lipid raft-associated protein, is involved in apical transport in MDCK cells. Proc. Natl. Acad. Sci. USA 96, 6241-6248.

Chih, B., Liu, P., Chinn, Y., Chalouni, C., Komuves, L. G., Hass, P. E., Sandoval, W. and Peterson, A. S. (2011). A ciliopathy complex at the transition zone protects the cilia as a privileged membrane domain. Nat. Cell Biol. 14, 61-72.

Das, A. and Guo, W. (2011). Rabs and the exocyst in ciliogenesis, tubulogenesis and beyond. Trends Cell Biol. 21, 383-386.

Finetti, F. and Baldari, C. T. (2013). Compartmentalization of signaling by vesicular trafficking: a shared building design for the immune synapse and the primary cilium. Immunol. Rev. 251, 97-112.

Follit, J. A., Tuft, R. A., Fogarty, K. E. and Pazour, G. J. (2006). The intraflagellar transport protein IFT20 is associated with the Golgi complex and is required for cilia assembly. Mol. Biol. Cell 17, 3781-3792.

Francis, S. S., Sfakianos, J., Lo, B. and Mellman, I. (2011). A hierarchy of signals regulates entry of membrane proteins into the ciliary membrane domain in epithelial cells. J. Cell Biol. 193, 219-233.

Gaus, K., Gratton, E., Kable, E. P. W., Jones, A. S., Gelissen, I., Kritharides, L. and Jessup, W. (2003). Visualizing lipid structure and raft domains in living cells with two-photon microscopy. Proc. Nat. Acad. Sci. USA 100, 15554-15559.

Gaus, K., Zech, T. and Harder, T. (2006). Visualizing membrane microdomains by Laurdan 2-photon microscopy. Mol. Membr. Biol. 23, 41-48.

Gerdes, J. M., Davis, E. E. and Katsanis, N. (2009). The vertebrate primary cilium in development, homeostasis, and disease. Cell 137, 32-45.

Goetz, S. C. and Anderson, K. V. (2010). The primary cilium: a signalling centre during vertebrate development. Nat. Rev. Genet. 11, 331-344.

Hehnly, H., Chen, C.-T., Powers, C. M., Liu, H.-L. and Doxsey, S. (2012). The centrosome regulates the Rab11- dependent recycling endosome pathway at appendages of the mother centriole. Curr. Biol. 22, 1944-1950.

Heider, M. R. and Munson, M. (2012). Exorcising the exocyst complex. Traffic 13, 898-907.
Hildebrandt, F. and Otto, E. (2005). Cilia and centrosomes: a unifying pathogenic concept for cystic kidney disease? Nat. Rev. Genet. 6, 928-940.

Hildebrandt, F., Benzing, T. and Katsanis, N. (2011). Ciliopathies. N. Engl. J. Med. 364, 1533-1543

Hu, Q., Milenkovic, L., Jin, H., Scott, M. P., Nachury, M. V., Spiliotis, E. T. and Nelson, W. J. (2010). A septin diffusion barrier at the base of the primary cilium maintains ciliary membrane protein distribution. Science 329, 436-439.

Ishikawa, H. and Marshall, W. F. (2011). Ciliogenesis: building the cell's antenna. Nat. Rev. Mol. Cell Biol. 12, 222-234.

Kee, H. L., Dishinger, J. F., Lynne Blasius, T., Liu, C.-J., Margolis, B. and Verhey, K. J. (2012). A size-exclusion permeability barrier and nucleoporins characterize a ciliary pore complex that regulates transport into cilia. Nat. Cell Biol. 14, 431-437.

Knodler, A., Feng, S., Zhang, J., Zhang, X., Das, A., Peränen, J. and Guo, W. (2010). Coordination of Rab8 and Rab11 in primary ciliogenesis. Proc. Natl. Acad. Sci. USA 107, 6346-6351

Kreitzer, G., Schmoranzer, J., Low, S. H., Li, X., Gan, Y., Weimbs, T., Simon, S. M. and Rodriguez-Boulan, E. (2003). Three-dimensional analysis of postGolgi carrier exocytosis in epithelial cells. Nat. Cell Biol. 5, 126-136.

Lim, Y. S., Chua, C. E. L. and Tang, B. L. (2011). Rabs and other small GTPases in ciliary transport. Biol. Cell 103, 209-221.

Lingwood, D. and Simons, K. (2010). Lipid rafts as a membrane-organizing principle. Science 327, 46-50.

Lisanti, M., Le Bivic, A., Saltiel, A. and Rodriguez-Boulan, E. (1990). Preferred apical distribution of glycosyl-phosphatidylinositol (GPI) anchored proteins: a highly conserved feature of the polarized epithelial cell phenotype. J. Mem. Biol. 113, 155-167.

Lisanti, M. and Rodriguez-Boulan, E. (1990). Glycophospholipid membrane anchoring provides clues to the mechanism of protein sorting in polarized epithelial cells. Trends Biochem. Sci. 15, 113-118.

Magal, L. G., Yaffe, Y., Shepshelovich, J., Aranda, J. F., de Marco, M. C., Gaus, K., Alonso, M. A. and Hirschberg, K. (2009). Clustering and lateral concentration of raft lipids by the MAL protein. Mol. Biol. Cell 20, 3751-3762.

Martin-Belmonte, F., Kremer, L., Albar, J., Marazuela, M. and Alonso, M. A (1998). Expression of the MAL gene in the thyroid: the MAL proteolipid, a component of glycolipid-enriched membranes, is apically distributed in thyroid follicles. Endocrinology 139, 2077-2084.

Meder, D., Shevchenko, A., Simons, K. and Füllekrug, J. (2005). Gp135 podocalyxin and NHERF-2 participate in the formation of a preapical domain during polarization of MDCK cells. J. Cell Biol. 168, 303-313.

Milenkovic, L., Scott, M. P. and Rohatgi, R. (2009). Lateral transport of Smoothened from the plasma membrane to the membrane of the cilium. J. Cell Biol. 187, 365-374. Millán, J. and Alonso, M. A. (1998). MAL, a novel integral membrane protein of human $\mathrm{T}$ lymphocytes, associates with glycosylphosphatidylinositol-anchored proteins and Src-like tyrosine kinases. Eur. J. Immunol. 28, 3675-3684.

Novarino, G., Akizu, N. and Gleeson, J. G. (2011). Modeling human disease in humans: the ciliopathies. Cell 147, 70-79.

Owen, D. M., Rentero, C., Magenau, A., Abu-Siniyeh, A. and Gaus, K. (2011). Quantitative imaging of membrane lipid order in cells and organisms. Nat. Protoc. 7 , 24-35.

Pazour, G. J., Dickert, B. L., Vucica, Y., Seeley, E. S., Rosenbaum, J. L., Witman, G. B. and Cole, D. G. (2000). Chlamydomonas IFT88 and Its mouse homologue, polycystic kidney disease gene $\mathrm{Tg} 737$, are required for assembly of cilia and flagella. J. Cell Biol. 151, 709-718

Puertollano, R., Martín-Belmonte, F., Millán, J., de Marco, M. C., Albar, J. P., Kremer, L. and Alonso, M. A. (1999). The MAL proteolipid is necessary for normal apical transport and accurate sorting of the influenza virus hemagglutinin in Madin-Darby canine kidney cells. J. Cell Biol. 145, 141-151.

Reiter, J. F. and Mostov, K. (2006). Vesicle transport, cilium formation, and membrane specialization: the origins of a sensory organelle. Proc. Natl. Acad. Sci. USA 103, 18383-18384

Rentero, C., Zech, T., Quinn, C. M., Engelhardt, K., Williamson, D., Grewal, T., Jessup, W., Harder, T. and Gaus, K. (2008). Functional implications of plasma membrane condensation for T cell activation. PLoS ONE 3, e2262.

Rosenbaum, J. L. and Witman, G. B. (2002). Intraflagellar transport. Nat. Rev. Mol. Cell Biol. 3, 813-825.

Salisbury, J. L., Suino, K. M., Busby, R. and Springett, M. (2002). Centrin-2 is required for centriole duplication in mammalian cells. Curr. Biol. 12, 1287-1292. Sorokin, S. (1962). Centrioles and the formation of rudimentary cilia by fibroblasts and smooth muscle cells. J. Cell Biol. 15, 363-377.

Takiar, V., Mistry, K., Carmosino, M., Schaeren-Wiemers, N. and Caplan, M. J. (2012). VIP17/MAL expression modulates epithelial cyst formation and ciliogenesis. Am. J. Physiol. Cell Physiol. 303, C862-C871.

Torkko, J. M., Manninen, A., Schuck, S. and Simons, K. (2008). Depletion of apical transport proteins perturbs epithelial cyst formation and ciliogenesis. J. Cell Sci. 121, 1193-1203.

Vieira, O. V., Verkade, P., Manninen, A. and Simons, K. (2005). FAPP2 is involved in the transport of apical cargo in polarized MDCK cells. J. Cell Biol. 170, 521-526. Vieira, O. V., Gaus, K., Verkade, P., Fullekrug, J., Vaz, W. L. C. and Simons, K. (2006). FAPP2, cilium formation, and compartmentalization of the apical 
membrane in polarized Madin-Darby canine kidney (MDCK) cells. Proc. Nat. Acad. Sci. USA 103, 18556-18561.

Wang, G., Krishnamurthy, K. and Bieberich, E. (2009). Regulation of primary cilia formation by ceramide. J. Lipid Res. 50, 2103-2110.

Watnick, T. and Germino, G. (2003). From cilia to cyst. Nat. Genet. 34, 355-356.

Westlake, C. J., Baye, L. M., Nachury, M. V., Wright, K. J., Ervin, K. E., Phu, L.,

Chalouni, C., Beck, J. S., Kirkpatrick, D. S., Slusarski, D. C. et al. (2011).

Primary cilia membrane assembly is initiated by Rab11 and transport protein particle II (TRAPPII) complex-dependent trafficking of Rabin8 to the centrosome. Proc. Natl. Acad. Sci. USA 108, 2759-2764.

Yoshimura, S.-i., Egerer, J., Fuchs, E., Haas, A. K. and Barr, F. A. (2007) Functional dissection of Rab GTPases involved in primary cilium formation. J. Cell Biol. 178, 363-369.

Zuo, X., Guo, W. and Lipschutz, J. H. (2009). The exocyst protein sec10 is necessary for primary ciliogenesis and cystogenesis in vitro. Mol. Biol. Cell 20, 2522-2529. 